\title{
New singularities in nonrelativistic coupled channel scattering. II. Fourth order
}

\author{
N. N. Khuri \\ Department of Physics, The Rockefeller University, New York, New York 10021 \\ Tai Tsun $\mathrm{Wu}$ \\ Gordon McKay Laboratory, Harvard University, Cambridge, Massachusetts 02138-2901 \\ and Theoretical Physics Division, CERN, CH-1211 Geneva 23, Switzerland
}

(Received 7 May 1997)

\begin{abstract}
We consider a two-channel nonrelativistic potential scattering problem, and study perturbation theory in fourth order for the forward amplitude. The main result is that the new singularity demonstrated in second order in the preceding paper I also occurs at the same point in fourth order. Its strength is again that of a pole. [S0556-2821(97)04023-X]
\end{abstract}

PACS number(s): 03.80.+r, 03.65.Nk

\section{INTRODUCTION}

In this paper we study the fourth-order forward scattering amplitude for the two-channel problem [1] posed in the preceding paper I. The purpose is to determine if the fourthorder amplitude has a singularity at the same point, $k=i\left(a^{2}+\mu^{2}\right) / 2 \mu$, and to see if this singularity is again a simple pole as in the second-order case. Our conclusion is indeed that the position of the singularity does not move in fourth order and has the strength of a simple pole.

In Sec. II, we start by writing down explicitly the fourthorder amplitude. It turns out to be similar to a three-loop vertex diagram in a three-dimensional quantum field theory. By a judicious choice of integration variables, we express this fourth-order forward amplitude as a two-dimensional integral with the integrand proportional to $(\widetilde{F})^{2}$, where $\widetilde{F}$ is the off-shell, nonforward, second-order amplitude.

The next step is thus to calculate $\widetilde{F}$ exactly, with full care about the $i \epsilon$ 's. In Sec. III, we will actually calculate a much more general result. Namely, we obtain the complete expression for the full Euclidean three-dimensional triangle diagram as a function of six variables. These are the three external $M$ 's and the three internal $m$ 's. The needed $\widetilde{F}$ can be obtained from this general result by analytic continuation in one of the internal masses.

In Sec. IV, we check the general result of Sec. III, and the analytic continuation, by calculating from it the elastic nonforward amplitude in second order and its forward limit. In addition, we introduce a method of approximating this amplitude near the forward direction and near the singularity at $k=i\left(a^{2}+\mu^{2}\right) / 2 \mu$.

In Sec. V, we analyze the fourth-order expression. The integral cannot be carried out exactly in terms of familiar functions. Instead, we introduce approximations in order to study the leading part of $\widetilde{F}$ near the forward direction and in the vicinity of the second-order singularity. We show that the singularity survives in $F^{(4)}$ with a nonvanishing residue.

\section{FOURTH-ORDER AMPLITUDE IN THE FORWARD DIRECTION}

We consider the two-coupled channel problem defined by the Hamiltonian $H$ : where $a$ is real and fixed, and $a^{2}$ is the energy difference between the two channels.

Since the potential has only off-diagonal elements, it is clear that $T_{11}^{(3)}=T_{22}^{(3)}=0$ for the third-order forward scattering amplitude. In fourth order, the forward amplitude $T_{22}^{(4)}$ is given by

$$
T_{22}^{(4)}(k)=-\frac{1}{\pi} F^{(4)}(k),
$$

where

$$
\begin{aligned}
F^{(4)}(k)= & \int d^{3} p_{1} \int d^{3} p_{2} \int d^{3} p_{3} \int d^{3} p_{4} \delta^{3}\left(\vec{p}_{1}+\vec{p}_{2}+\vec{p}_{3}\right. \\
& \left.+\vec{p}_{4}\right) \frac{1}{\left(\vec{k}+\vec{p}_{1}\right)^{2}-\left(k^{2}+a^{2}\right)-i \epsilon} \\
& \times \frac{1}{\left(\vec{k}+\vec{p}_{1}+\vec{p}_{2}\right)^{2}-k^{2}-i \epsilon} \\
& \times \frac{1}{\left(\vec{k}-\vec{p}_{4}\right)^{2}-\left(k^{2}+a^{2}\right)-i \epsilon} \prod_{j=1}^{4} \frac{1}{\left(p_{j}^{2}+\mu^{2}\right)}
\end{aligned}
$$

This integral is represented by the diagram given in Fig. 1.

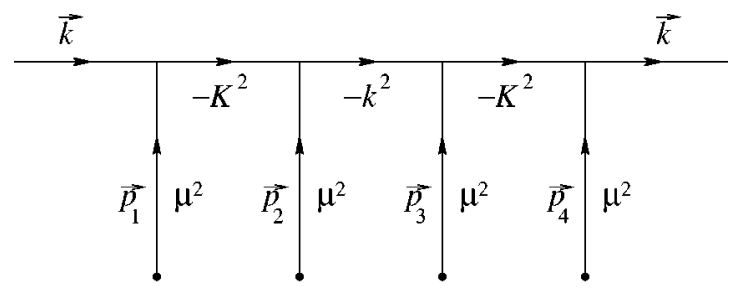

FIG. 1. Schematic representation of the fourth-order amplitude. Note the horizontal inner lines have an imaginary mass. 
The graph in Fig. 1 has a symmetry when cut by a vertical line in the middle. This suggests using $\left(\vec{p}_{1}+\vec{p}_{2}\right)$ as a variable of integration. We set

$$
\vec{\Delta}=\vec{p}_{1}+\vec{p}_{2}=-\left(\vec{p}_{3}+\vec{p}_{4}\right)
$$

From Eq. (2.3) with $\vec{p}_{4}^{\prime}=-\vec{p}_{4}$, we have

$$
\begin{aligned}
F^{(4)}(k)= & \int d^{3} p_{1} \int d^{3} \Delta \int d^{3} p_{4}^{\prime} \frac{1}{\left(\vec{k}+\vec{p}_{1}\right)^{2}-K^{2}-i \epsilon} \frac{1}{(\vec{\Delta}+\vec{k})^{2}-k^{2}-i \epsilon} \frac{1}{\left(\vec{k}+\vec{p}_{4}\right)^{2}-K^{2}-i \epsilon} \\
& \times \frac{1}{p_{1}^{2}+\mu^{2}} \frac{1}{\left(\vec{p}_{1}-\vec{\Delta}\right)^{2}+\mu^{2}} \frac{1}{p_{4}^{\prime}{ }^{2}+\mu^{2}} \frac{1}{\left(\vec{p}_{4}{ }^{\prime}-\vec{\Delta}\right)^{2}+\mu^{2}}=\int d^{3} \Delta \frac{1}{(\vec{k}+\vec{\Delta})^{2}-k^{2}-i \epsilon}[\widetilde{F}(\vec{\Delta})]^{2},
\end{aligned}
$$

where

$$
\widetilde{F}(\vec{\Delta}) \equiv \int d^{3} p \frac{1}{(\vec{k}+\vec{p})^{2}-K^{2}-i \epsilon} \cdot \frac{1}{p^{2}+\mu^{2}} \cdot \frac{1}{(\vec{p}-\vec{\Delta})^{2}+\mu^{2}}
$$

Here $\widetilde{F}(\vec{\Delta})$ is a function of $\Delta^{2}$ and $\vec{k} \cdot \vec{\Delta}$, and as in paper I, Sec. III, $K^{2}=k^{2}+a^{2}$. $\widetilde{F}$ is essentially the nonforward, offshell, second-order amplitude. Similar to the $F$ studied in the previous paper, it will turn out to be an elementary function involving only logarithms and square roots. As $\vec{\Delta} \rightarrow 0$, $\widetilde{F} \rightarrow F(K)$, the second-order amplitude given in Eq. (4.7) of paper I. Thus, for $\vec{\Delta}=0, \widetilde{F}$ has poles at $k=+i\left(a^{2}+\mu^{2}\right) / 2 \mu$, and the integration over $\Delta^{2}$ near $\Delta^{2} \approx 0$ is likely to produce a singularity in $F^{(4)}$ at $k=+i\left(a^{2}+\mu^{2}\right) / 2 \mu$. One can get a confirmation of this intuitive hint by using standard parametric techniques to study the singularities of $F^{(4)}$.

We redraw the diagram in Fig. 1 as shown in Fig. 2. $F^{(4)}(k)$ is thus given by a three-loop vertex diagram with four Euclidean internal lines of mass $\mu^{2}$ and three (horizontal) internal lines representing non-Euclidean propagators.

We proceed in the next section to calculate $\widetilde{F}(\vec{\Delta})$ exactly. Actually, we will first carry out a more general calculation and then obtain $\widetilde{F}$ by going to a special case and carrying out an analytic continuation. The purpose is to calculate the second-order perturbation in a symmetrical way.

\section{TRIANGLE DIAGRAM IN THREE DIMENSIONS}

In Eq. (2.5), the on-shell scattering amplitude in the fourth order is expressed as an integral of the square of the off-

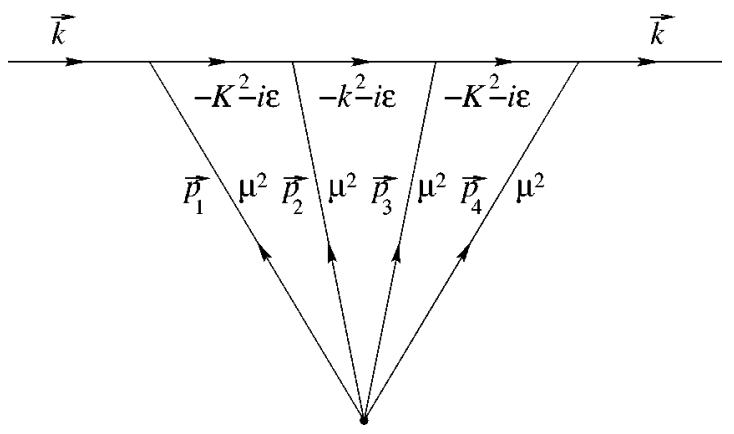

FIG. 2. Three-loop vertex diagram corresponding to the fourthorder amplitude, $\vec{p}_{1}+\vec{p}_{2}+\vec{p}_{3}+\vec{p}_{4}=0$. shell, nonforward scattering amplitude $\widetilde{F}$ in the second order.

\section{A. Permutation symmetry}

For technical reasons, it is convenient to study the Euclidean version of a generalization of $\widetilde{F}$. More precisely, the integral to be studied in this section comes from the triangle diagram in three dimensions [2], as shown in Fig. 3:

$$
F=\int_{0}^{1} d \alpha_{1} d \alpha_{2} d \alpha_{3} \delta\left(1-\alpha_{1}-\alpha_{2}-\alpha_{3}\right) D^{-3 / 2},
$$

where

$$
D=M_{1}^{2} \alpha_{2} \alpha_{3}+M_{2}^{2} \alpha_{3} \alpha_{1}+M_{3}^{2} \alpha_{1} \alpha_{2}+\alpha_{1} m_{1}^{2}+\alpha_{2} m_{2}^{2}+\alpha_{3} m_{3}^{2} .
$$

This shows explicitly the symmetry under the permutation group for 1,2 , and 3 . It is also assumed that the three positive numbers $M_{1}, M_{2}$, and $M_{3}$ form a real triangle, i.e., $M_{1}+M_{2}>M_{3}>0$, etc. The appearance of the power $-3 / 2$ in Eq. (3.1) is related to the dimensionality being 3, not 4.

The key idea of the present calculation is to maintain this permutation symmetry as much as possible.

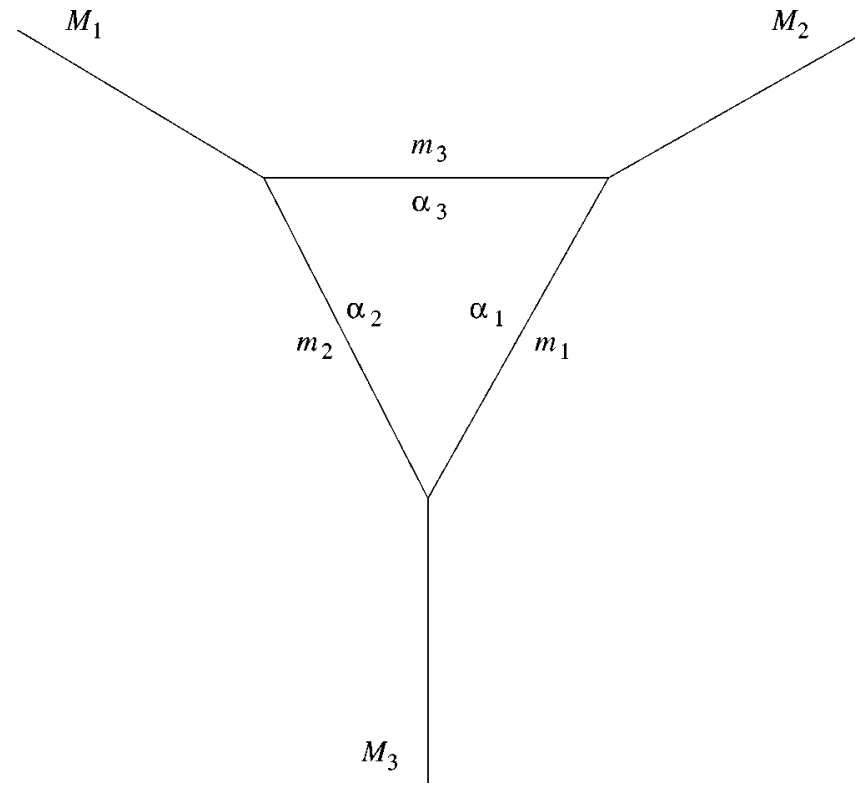

FIG. 3. The triangle diagram. 
Because of the presence of the $\delta$ function in the integrand, the right-hand side of Eq. (3.1) is effectively a twodimensional integral. As a first example of using the permutation symmetry, consider the rotation

$$
\left(\begin{array}{l}
w \\
u \\
v
\end{array}\right)=\left(\begin{array}{ccc}
\frac{1}{\sqrt{3}} & \frac{1}{\sqrt{3}} & \frac{1}{\sqrt{3}} \\
-\frac{1}{\sqrt{6}} & -\frac{1}{\sqrt{6}} & \sqrt{\frac{2}{3}} \\
\frac{1}{\sqrt{2}} & -\frac{1}{\sqrt{2}} & 0
\end{array}\right)\left(\begin{array}{l}
\alpha_{1} \\
\alpha_{2} \\
\alpha_{3}
\end{array}\right) .
$$

Because of the $\delta$ function, $w=1 / \sqrt{3}$, and hence

$$
\begin{gathered}
\alpha_{1}=-\frac{1}{\sqrt{6}} u+\frac{1}{\sqrt{2}} v+\frac{1}{3}, \\
\alpha_{2}=-\frac{1}{\sqrt{6}} u-\frac{1}{\sqrt{2}} v+\frac{1}{3}, \\
\alpha_{3}=\sqrt{\frac{2}{3}} u+\frac{1}{3} .
\end{gathered}
$$

In terms of $u$ and $v$, the $D$ of Eq. (3.2) is

$$
\begin{aligned}
D= & \frac{-2 M_{1}^{2}-2 M_{2}^{2}+M_{3}^{2}}{6} u^{2}-\frac{M_{1}^{2}-M_{2}^{2}}{\sqrt{3}} u v-\frac{M_{3}^{2}}{2} v^{2} \\
& +\frac{1}{3 \sqrt{6}}\left[M_{1}^{2}+M_{2}^{2}-2 M_{3}^{2}-3\left(m_{1}^{2}+m_{2}^{2}-2 m_{3}^{2}\right)\right] u \\
& -\frac{1}{3 \sqrt{2}}\left[M_{1}^{2}-M_{2}^{2}-3\left(m_{1}^{2}-m_{2}^{2}\right)\right] v \\
& +\frac{1}{9}\left[M_{1}^{2}+M_{2}^{2}+M_{3}^{2}+3\left(m_{1}^{2}+m_{2}^{2}+m_{3}^{2}\right)\right] .
\end{aligned}
$$

As seen from Eqs. (3.4) and (3.5), $\alpha_{3}$ and hence $M_{3}$ take a slightly different role than the corresponding formulas with indices 1 and 2. In order to recover the full permutation symmetry, consider the quadratic part of Eq. (3.5) as given by the matrix

$$
\mathcal{M}=\left(\begin{array}{cc}
\frac{2 M_{1}^{2}+2 M_{2}^{2}-M_{3}^{2}}{6} & \frac{M_{1}^{2}-M_{2}^{2}}{2 \sqrt{3}} \\
\frac{M_{1}^{2}-M_{2}^{2}}{2 \sqrt{3}} & \frac{M_{3}^{2}}{2}
\end{array}\right) .
$$

The determinant of this $2 \times 2$ matrix is fully symmetrical under the permutation group

$$
\operatorname{det} \mathcal{M}=\frac{1}{12}(-A+2 B),
$$

where

$$
A=M_{1}^{4}+M_{2}^{4}+M_{3}^{4}, \quad B=M_{1}^{2} M_{2}^{2}+M_{2}^{2} M_{3}^{2}+M_{3}^{2} M_{1}^{2} .
$$

Since $M_{1}, M_{2}$, and $M_{3}$ form a real triangle, it follows that

$$
2 B>A>B,
$$

and in particular $\operatorname{det} \mathcal{M}>0$.

Furthermore, the two eigenvalues of this $2 \times 2$ matrix $\mathcal{M}$ are given by

$$
\begin{aligned}
& \lambda_{+}=\frac{1}{6}\left[M_{1}^{2}+M_{2}^{2}+M_{3}^{2}+2 \sqrt{A-B}\right], \\
& \lambda_{-}=\frac{1}{6}\left[M_{1}^{2}+M_{2}^{2}+M_{3}-2 \sqrt{A-B}\right],
\end{aligned}
$$

and hence are also fully symmetrical. Note that, by Eqs. (3.8), $\lambda_{+}>\lambda_{-}>0$.

The absence of full permutation symmetry occurs in the eigenvectors. Since the index 3 has been treated differently from the indices 1 and 2, we define $\theta_{3}$ by

$$
\mathcal{M}\left(\begin{array}{c}
\cos \theta_{3} \\
\sin \theta_{3}
\end{array}\right)=\lambda_{+}\left(\begin{array}{c}
\cos \theta_{3} \\
\sin \theta_{3}
\end{array}\right), \quad \mathcal{M}\left(\begin{array}{c}
-\sin \theta_{3} \\
\cos \theta_{3}
\end{array}\right)=\lambda_{-}\left(\begin{array}{c}
-\sin \theta_{3} \\
\cos \theta_{3}
\end{array}\right) .
$$

Since the overall signs of the eigenvectors are not defined, $\theta_{3}$ has an ambiguity of $180^{\circ}$. It is thus advantageous to work with $\tan \theta_{3}$ instead.

By permutation, $\theta_{1}$ and $\theta_{2}$ can be defined in a similar manner. The results are explicitly

$$
\begin{gathered}
\theta_{1}=\theta_{3}-\frac{2 \pi}{3}, \\
\theta_{2}=\theta_{1}-\frac{2 \pi}{3}, \\
\theta_{3}=\theta_{2}-\frac{2 \pi}{3}+2 \pi, \\
\tan \theta_{1}=\sqrt{3} \frac{M_{2}^{2}-M_{3}^{2}}{-2 M_{1}^{2}+M_{2}^{2}+M_{3}^{2}+2 \sqrt{A-B}}, \\
\tan \theta_{3}=\sqrt{3} \frac{M_{3}^{2}-M_{1}^{2}}{M_{1}^{2}-2 M_{2}^{2}+M_{3}^{2}+2 \sqrt{A-B}} \\
M_{1}^{2}+M_{2}^{2}-2 M_{3}^{2}+2 \sqrt{A-B}
\end{gathered}
$$

In this way, the permutation symmetry is fully recovered. As seen in the development of this section, Sec. III A, the permutation symmetry is maintained as much as possible in the sense that, while it cannot be kept in every equation, it is restored at the end of each major step. 


\section{B. Canonical form for $D$}

Since the quantity $D$ of Eq. (3.2) appears in the integrand of $F$ as given by Eq. (3.1), it is essential to recast this $D$ in a convenient form. From Eq. (3.5), a tedious but straightforward calculation gives

$$
\begin{aligned}
D= & \frac{-2 M_{1}^{2}-2 M_{2}^{2}+M_{3}^{2}}{6}(u-\bar{u})^{2} \\
& -\frac{M_{1}^{2}-M_{2}^{2}}{\sqrt{3}}(u-\bar{u})(v-\bar{v})-\frac{M_{3}^{2}}{2}(v-\bar{v})^{2}+\bar{D},
\end{aligned}
$$

where

$$
\begin{aligned}
\bar{u}= & \frac{1}{\sqrt{6}(-A+2 B)}\left\{M_{1}^{4}+M_{2}^{4}-2 M_{3}^{4}+M_{2}^{2} M_{3}^{2}+M_{3}^{2} M_{1}^{2}\right. \\
& -2 M_{1}^{2} M_{2}^{2}-3\left[M_{3}^{2}\left(m_{1}^{2}+m_{2}^{2}-2 m_{3}^{2}\right)\right. \\
& \left.\left.+\left(M_{1}^{2}-M_{2}^{2}\right)\left(m_{1}^{2}-m_{2}^{2}\right)\right]\right\} \\
\bar{v}= & \frac{1}{\sqrt{2}(-A+2 B)}\left\{-\left(M_{1}^{2}-M_{2}^{2}\right)\left(M_{1}^{2}+M_{2}^{2}-M_{3}^{2}\right)\right. \\
& +\left[\left(M_{1}^{2}-M_{2}^{2}\right)\left(m_{1}^{2}+m_{2}^{2}-2 m_{3}^{2}\right)\right. \\
& \left.\left.+\left(2 M_{1}^{2}+2 M_{2}^{2}-M_{3}^{2}\right)\left(m_{1}^{2}-m_{2}^{2}\right)\right]\right\}
\end{aligned}
$$

and

$$
\begin{aligned}
\bar{D}= & \frac{1}{-M_{1}^{4}-M_{2}^{4}-M_{3}^{4}+2 M_{2}^{2} M_{3}^{2}+2 M_{3}^{2} M_{1}^{2}+2 M_{1}^{2} M_{2}^{2}}\left\{M_{1}^{2} M_{2}^{2} M_{3}^{2}+m_{1}^{2} M_{1}^{2}\left(-M_{1}^{2}+M_{2}^{2}+M_{3}^{2}\right)+m_{2}^{2} M_{2}^{2}\left(M_{1}^{2}-M_{2}^{2}+M_{3}^{2}\right)\right. \\
& \left.+m_{3}^{2} M_{3}^{2}\left(M_{1}^{2}+M_{2}^{2}-M_{3}^{2}\right)+M_{1}^{2}\left(m_{1}^{2}-m_{2}^{2}\right)\left(m_{1}^{2}-m_{3}^{2}\right)+M_{2}^{2}\left(m_{2}^{2}-m_{3}^{2}\right)\left(m_{2}^{2}-m_{1}^{2}\right)+M_{3}^{2}\left(m_{3}^{2}-m_{1}^{2}\right)\left(m_{3}^{2}-m_{2}^{2}\right)\right\} .
\end{aligned}
$$

It follows from Eqs. (3.4) and (3.14) that the values of $\bar{\alpha}_{1}, \bar{\alpha}_{2}$, and $\bar{\alpha}_{3}$ corresponding to $\bar{u}$ and $\bar{v}$ are explicitly given by

$$
\begin{aligned}
& \bar{\alpha}_{1}=\frac{M_{1}^{2}\left(M_{2}^{2}+M_{3}^{2}-M_{1}^{2}\right)-M_{1}^{2}\left(m_{2}^{2}+m_{3}^{2}-2 m_{1}^{2}\right)-\left(M_{2}^{2}-M_{3}^{2}\right)\left(m_{2}^{2}-m_{3}^{2}\right)}{-M_{1}^{4}-M_{2}^{4}-M_{3}^{4}+2 M_{2}^{2} M_{3}^{2}+2 M_{3}^{2} M_{1}^{2}+2 M_{1}^{2} M_{2}^{2}}, \\
& \bar{\alpha}_{2}=\frac{M_{2}^{2}\left(M_{3}^{2}+M_{1}^{2}-M_{2}^{2}\right)-M_{2}^{2}\left(m_{3}^{2}+m_{1}^{2}-2 m_{2}^{2}\right)-\left(M_{3}^{2}-M_{1}^{2}\right)\left(m_{3}^{2}-m_{1}^{2}\right)}{-M_{1}^{4}-M_{2}^{4}-M_{3}^{4}+2 M_{2}^{2} M_{3}^{2}+2 M_{3}^{2} M_{1}^{2}+2 M_{1}^{2} M_{2}^{2}}, \\
& \bar{\alpha}_{3}=\frac{M_{3}^{2}\left(M_{1}^{2}+M_{2}^{2}-M_{3}^{2}\right)-M_{3}^{2}\left(m_{1}^{2}+m_{2}^{2}-2 m_{3}^{2}\right)-\left(M_{1}^{2}-M_{2}^{2}\right)\left(m_{1}^{2}-m_{2}^{2}\right)}{-M_{1}^{4}-M_{2}^{4}-M_{3}^{4}+2 M_{2}^{2} M_{3}^{2}+2 M_{3}^{2} M_{1}^{2}+2 M_{1}^{2} M_{2}^{2}} .
\end{aligned}
$$

These formulas all respect permutation symmetry.

With reference to Eq. (3.13), it still remains to determine a pair of variables, say, $x$ and $y$, that, from the point of view of permutation symmetry, are superior to the $u$ and $v$. This can be accomplished by making use of the simple relations between the $\theta_{1}, \theta_{2}$, and $\theta_{3}$, as given by Eq. (3.12). By Eq. (3.11), $\mathcal{M}$ can be expressed as

$$
\mathcal{M}=\left(\begin{array}{cc}
\cos \theta_{3} & -\sin \theta_{3} \\
\sin \theta_{3} & \cos \theta_{3}
\end{array}\right)\left(\begin{array}{cc}
\lambda_{+} & 0 \\
0 & \lambda_{-}
\end{array}\right)\left(\begin{array}{cc}
\cos \theta_{3} & \sin \theta_{3} \\
-\sin \theta_{3} & \cos \theta_{3}
\end{array}\right)
$$

and hence the $D$ of Eq. (3.13) is

$$
\begin{aligned}
D= & -u-\bar{u} v-\bar{v}\left(\begin{array}{cc}
\cos \theta_{3} & -\sin \theta_{3} \\
\sin \theta_{3} & \cos \theta_{3}
\end{array}\right)\left(\begin{array}{cc}
\lambda_{+} & 0 \\
0 & \lambda_{-}
\end{array}\right) \\
& \times\left(\begin{array}{cc}
\cos \theta_{3} & \sin \theta_{3} \\
-\sin \theta_{3} & \cos \theta_{3}
\end{array}\right)\left(\begin{array}{l}
u-\bar{u} \\
v-\bar{v}
\end{array}\right)+\bar{D} .
\end{aligned}
$$

Since $\lambda_{+}$and $\lambda_{-}$are both positive, it is natural therefore to define

$$
\left(\begin{array}{l}
x \\
y
\end{array}\right)=\left(\begin{array}{cc}
\sqrt{\lambda_{+}} & 0 \\
0 & \sqrt{\lambda_{-}}
\end{array}\right)\left(\begin{array}{cc}
\cos \theta_{3} & \sin \theta_{3} \\
-\sin \theta_{3} & \cos \theta_{3}
\end{array}\right)\left(\begin{array}{c}
u-\bar{u} \\
v-\bar{v}
\end{array}\right),
$$

and the desired canonical form for $D$ is

$$
D=-x^{2}-y^{2}-\bar{D},
$$

where $\bar{D}$ is given by Eq. (3.15).

\section{Auxiliary triangular region $\bar{R}$}

It is useful to define a region $\bar{R}$ by

$$
\bar{\alpha}_{1}>0, \quad \bar{\alpha}_{2}>0, \quad \bar{\alpha}_{3}>0
$$

It is seen from Eq. (3.16) that these $\bar{\alpha}_{j}$ are all linear in $m_{1}^{2}$, $m_{2}^{2}$, and $m_{3}^{2}$. Therefore, for given values of $M_{1}^{2}, M_{2}^{2}$, and $M_{3}^{2}$, $\bar{R}$ is a triangle in the plane with the variables

$$
\bar{m}_{1}^{2}=m_{1}^{2}-m_{0}^{2}, \quad \bar{m}_{2}^{2}=m_{2}^{2}-m_{0}^{2}, \quad \bar{m}_{3}^{2}=m_{3}^{2}-m_{0}^{2},
$$


where

$$
m_{0}^{2}=\frac{1}{3}\left(m_{1}^{2}+m_{2}^{2}+m_{3}^{2}\right)
$$

These $\bar{m}$ 's satisfy $\bar{m}_{1}^{2}+\bar{m}_{2}^{2}+\bar{m}_{3}^{2}=0$. In this $\bar{m}_{1}^{2}, \bar{m}_{2}^{2}, \bar{m}_{3}^{2}$ plane, the three sides of the triangle are given by the straight lines $L_{1}: \bar{\alpha}_{1}=0 ; L_{2}: \bar{\alpha}_{2}=0 ;$ and $L_{3}: \bar{\alpha}_{3}=0$. Since $\bar{\alpha}_{1}+\bar{\alpha}_{2}+\bar{\alpha}_{3}=1$, the triangle has a positive area. The three vertices of this triangle are given explicitly by

$$
\begin{aligned}
& \bar{P}_{1}\left(\frac{1}{3}\left(M_{2}^{2}+M_{3}^{2}\right), \frac{1}{3}\left(M_{2}^{2}-2 M_{3}^{2}\right), \frac{1}{3}\left(-2 M_{2}^{2}+M_{3}^{2}\right)\right), \\
& \bar{P}_{2}\left(\frac{1}{3}\left(-2 M_{3}^{2}+M_{1}^{2}\right), \frac{1}{3}\left(M_{3}^{2}+M_{1}^{2}\right), \frac{1}{3}\left(M_{3}^{2}-2 M_{1}^{2}\right)\right), \\
& \bar{P}_{3}\left(\frac{1}{3}\left(M_{1}^{2}-2 M_{2}^{2}\right), \frac{1}{3}\left(-2 M_{1}^{2}+M_{2}^{2}\right), \frac{1}{3}\left(M_{1}^{2}+M_{2}^{2}\right)\right),
\end{aligned}
$$

in the $\bar{m}_{1}^{2}, \bar{m}_{2}^{2}, \bar{m}_{3}^{2}$ plane, where $\bar{P}_{1}$, for example, is the point of intersection of $L_{2}$ and $L_{3}$. Note that this $\bar{P}_{1}$ does not depend on $M_{1}$. A set of values $\left(M_{1}^{2}, M_{2}^{2}, M_{3}^{2}, m_{1}^{2}, m_{2}^{2}, m_{3}^{2}\right)$ is in $\bar{R}$ if and only if

$$
\left(\bar{m}_{1}^{2}, \bar{m}_{2}^{2}, \bar{m}_{3}^{2}\right) \text { is inside the triangle } \Delta \bar{P}_{1} \bar{P}_{2} \bar{P}_{3} \text {. }
$$

The auxiliary region $\bar{R}$ is useful for the following reason. In the evaluation of $F$ as given by Eq. (3.1), it is necessary to be very careful about signs. Permutation symmetry can be applied in a straightforward way when the set of values $\left(M_{1}^{2}, M_{2}^{2}, M_{3}^{2}, m_{1}^{2}, m_{2}^{2}, m_{3}^{2}\right)$ is inside this region $\bar{R}$. Accordingly, we shall first evaluate $F$ for values inside $\bar{R}$, and then apply analytic continuation to extend the result beyond $\bar{R}$. The necessary analytic continuation is essentially trivial.

\section{Triangular region of integration}

So far we have worked only with the function $D$ of Eq. (3.2). Attention is now shifted to the integral $F$ of Eq. (3.1). Let $R$ be the region in the $(x, y)$ plane, defined by Eq. (3.19), where

$$
\alpha_{1}>0, \quad \alpha_{2}>0, \quad \text { and } \quad \alpha_{3}>0
$$

With the $A$ and $B$ defined by Eq. (3.8), this $F$ is

$$
F=2(-A+2 B)^{-1 / 2} \bar{F},
$$

where

$$
\bar{F}=\int_{R} d x d y D^{-3 / 2}=\int_{R} d x d y\left(-x^{2}-y^{2}+\bar{D}\right)^{-3 / 2} \text {. }
$$

In order to evaluate $\bar{F}$, the first step is to express the inequalities (3.25) in terms of $x$ and $y$. This is straightforward and the result is

$$
\cos \theta_{1} \frac{x}{\sqrt{\lambda_{+}}}-\sin \theta_{1} \frac{y}{\sqrt{\lambda_{-}}}>-\sqrt{\frac{3}{2}} \bar{\alpha}_{1}
$$

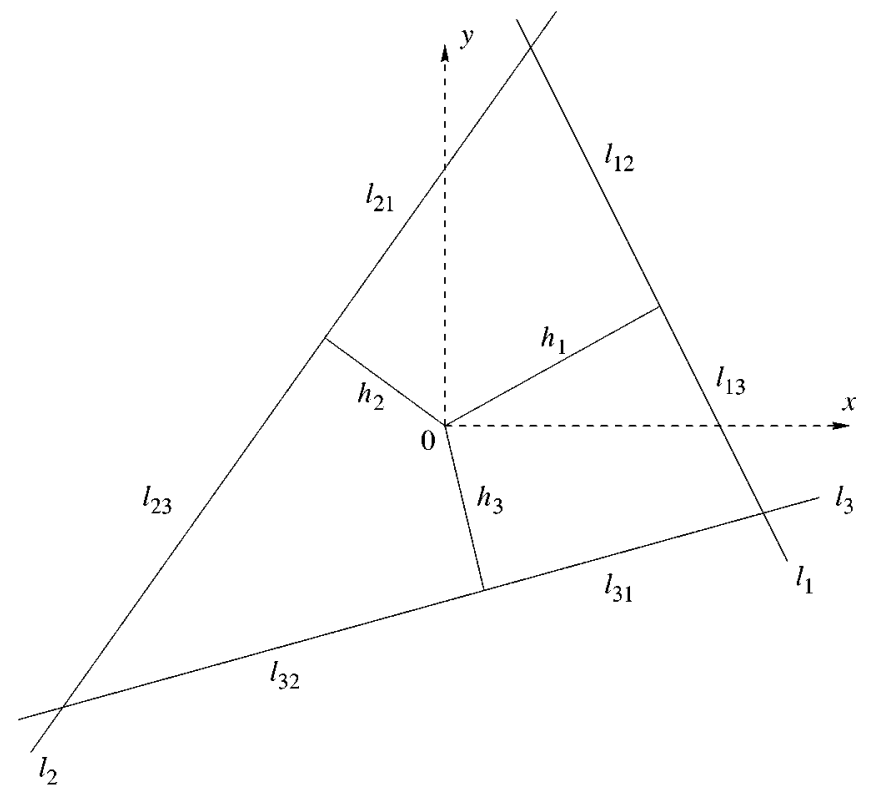

FIG. 4. The region of integration $R$, which is inside the triangle defined by the straight lines $l_{1}, l_{2}$, and $l_{3}$.

$$
\begin{gathered}
\cos \theta_{2} \frac{x}{\sqrt{\lambda_{+}}}-\sin \theta_{2} \frac{y}{\sqrt{\lambda_{-}}}>-\sqrt{\frac{3}{2}} \bar{\alpha}_{2}, \\
\cos \theta_{3} \frac{x}{\sqrt{\lambda_{+}}}-\sin \theta_{3} \frac{y}{\sqrt{\lambda_{-}}}>-\sqrt{\frac{3}{2}} \bar{\alpha}_{3},
\end{gathered}
$$

where the various quantities are given by Eqs. (3.10), (3.12), and (3.16). The geometry is shown in Fig. 4, where the three straight lines $\ell_{1}, \ell_{2}$, and $\ell_{3}$ are given by Eq. (3.28) with $>$ replaced by $=$. In Fig. 4 , the $h_{1}, h_{2}$, and $h_{3}$ are the distances between the origin $O$ and the $\ell_{1}, \ell_{2}$, and $\ell_{3}$, respectively. The six lengths $\ell_{12}, \ell_{23}, \ell_{31}, \ell_{21}, \ell_{13}$, and $\ell_{32}$ are the distances between the vertices of the triangle and the points where the perpendiculars meet the sides. The convention is used that all these nine lengths are taken to be positive if the geometry is as shown in the figure. They can take on negative values by analytic continuation.

The quantities, $h_{1}, h_{2}$, and $h_{3}$ are the easiest ones to obtain. They are given by

$$
\begin{aligned}
& h_{1}=\frac{\sqrt{\frac{3}{2}} \bar{\alpha}_{1}}{\sqrt{\frac{\cos ^{2} \theta_{1}}{\lambda_{+}}+\frac{\sin ^{2} \theta_{1}}{\lambda_{-}}}}, \\
& h_{2}=\frac{\sqrt{\frac{3}{2}} \bar{\alpha}_{2}}{\sqrt{\frac{\cos ^{2} \theta_{2}}{\lambda_{+}}+\frac{\sin ^{2} \theta_{2}}{\lambda_{-}}}}, \\
& h_{3}=\frac{\sqrt{\frac{3}{2}} \bar{\alpha}_{3}}{\sqrt{\frac{\cos ^{2} \theta_{3}}{\lambda_{+}}+\frac{\sin ^{2} \theta_{3}}{\lambda_{-}}}} .
\end{aligned}
$$

These formulas have the important implication that $h_{1}, h_{2}$, and $h_{3}$ have the same sign, i.e., positive, if and only if the 
inequalities (3.21) are satisfied. In other words, they are all positive as shown in Fig. 4 if and only if the set of parameters $\left(M_{1}^{2}, M_{2}^{2}, M_{3}^{2}, m_{1}^{2}, m_{2}^{2}, m_{3}^{2}\right)$ is in the auxiliary region $\bar{R}$. This is the reason for the detailed description of $\bar{R}$ given in Sec. III C.

The corresponding formulas for the $\ell_{i j}$ 's are more complicated:

$$
\begin{aligned}
& \ell_{12}=\frac{h_{2}-h_{1} \cos \left(\theta_{1}^{\prime}-\theta_{2}^{\prime}\right)}{\sin \left(\theta_{1}^{\prime}-\theta_{2}^{\prime}\right)}, \quad \ell_{21}=\frac{h_{1}-h_{2} \cos \left(\theta_{1}^{\prime}-\theta_{2}^{\prime}\right)}{\sin \left(\theta_{1}^{\prime}-\theta_{2}^{\prime}\right)}, \\
& \ell_{23}=\frac{h_{3}-h_{2} \cos \left(\theta_{2}^{\prime}-\theta_{3}^{\prime}\right)}{\sin \left(\theta_{2}^{\prime}-\theta_{3}^{\prime}\right)}, \quad \ell_{32}=\frac{h_{2}-h_{3} \cos \left(\theta_{2}^{\prime}-\theta_{3}^{\prime}\right)}{\sin \left(\theta_{2}^{\prime}-\theta_{3}^{\prime}\right)}, \\
& \ell_{31}=\frac{h_{1}-h_{3} \cos \left(\theta_{3}^{\prime}-\theta_{1}^{\prime}\right)}{\sin \left(\theta_{3}^{\prime}-\theta_{1}^{\prime}\right)}, \quad \ell_{13}=\frac{h_{3}-h_{1} \cos \left(\theta_{3}^{\prime}-\theta_{1}^{\prime}\right)}{\sin \left(\theta_{3}^{\prime}-\theta_{1}^{\prime}\right)},
\end{aligned}
$$

where the angles $\theta_{1}^{\prime}, \theta_{2}^{\prime}$, and $\theta_{3}^{\prime}$ are given by

$$
\begin{aligned}
& \cos \theta_{1}^{\prime}=\frac{\frac{\cos \theta_{1}}{\sqrt{\lambda_{+}}}}{\sqrt{\frac{\cos ^{2} \theta_{1}}{\lambda_{+}}+\frac{\sin ^{2} \theta_{1}}{\lambda_{-}}}}, \quad \sin \theta_{1}^{\prime}=\frac{\frac{\sin \theta_{1}}{\sqrt{\lambda_{-}}}}{\sqrt{\frac{\cos ^{2} \theta_{1}}{\lambda_{+}}+\frac{\sin ^{2} \theta_{1}}{\lambda_{-}}}}, \\
& \cos \theta_{2}^{\prime}=\frac{\frac{\cos \theta_{2}}{\sqrt{\lambda_{+}}}}{\sqrt{\frac{\cos ^{2} \theta_{2}}{\lambda_{+}}+\frac{\sin ^{2} \theta_{2}}{\lambda_{-}}}}, \quad \sin \theta_{2}^{\prime}=\frac{\frac{\sin \theta_{2}}{\sqrt{\lambda_{-}}}}{\sqrt{\frac{\cos ^{2} \theta_{2}}{\lambda_{+}}+\frac{\sin ^{2} \theta_{2}}{\lambda_{-}}}}, \\
& \cos \theta_{3}^{\prime}=\frac{\frac{\cos \theta_{3}}{\sqrt{\lambda_{+}}}}{\sqrt{\frac{\cos ^{2} \theta_{3}}{\lambda_{+}}+\frac{\sin ^{2} \theta_{3}}{\lambda_{-}}}}, \quad \sin \theta_{3}^{\prime}=\frac{\frac{\sin \theta_{3}}{\sqrt{\lambda_{-}}}}{\sqrt{\frac{\cos ^{2} \theta_{3}}{\lambda_{+}}+\frac{\sin ^{2} \theta_{3}}{\lambda_{-}}}},
\end{aligned}
$$

where the square roots in the denominators are always taken to be positive so that $\theta_{j}^{\prime} \rightarrow \theta_{j}$ for $j=1,2,3$, when $\lambda_{+} \rightarrow \lambda_{-}$.

\section{E. Integration}

With this setup, it is natural to split the region $R$ of integration into six pieces. These six pieces are related to each other through the permutation group for $(1,2,3)$. With reference to Fig. 5, where one of six subregions is shown shaded, define

$$
\bar{F}_{12}=\int_{\text {shaded region }} d x d y\left(-x^{2}-y^{2}+\bar{D}\right)^{-3 / 2}
$$

Then

$$
\bar{F}=\sum_{\substack{\text { six elements of } \\ \text { permutation group }}} \bar{F}_{12} \text {. }
$$

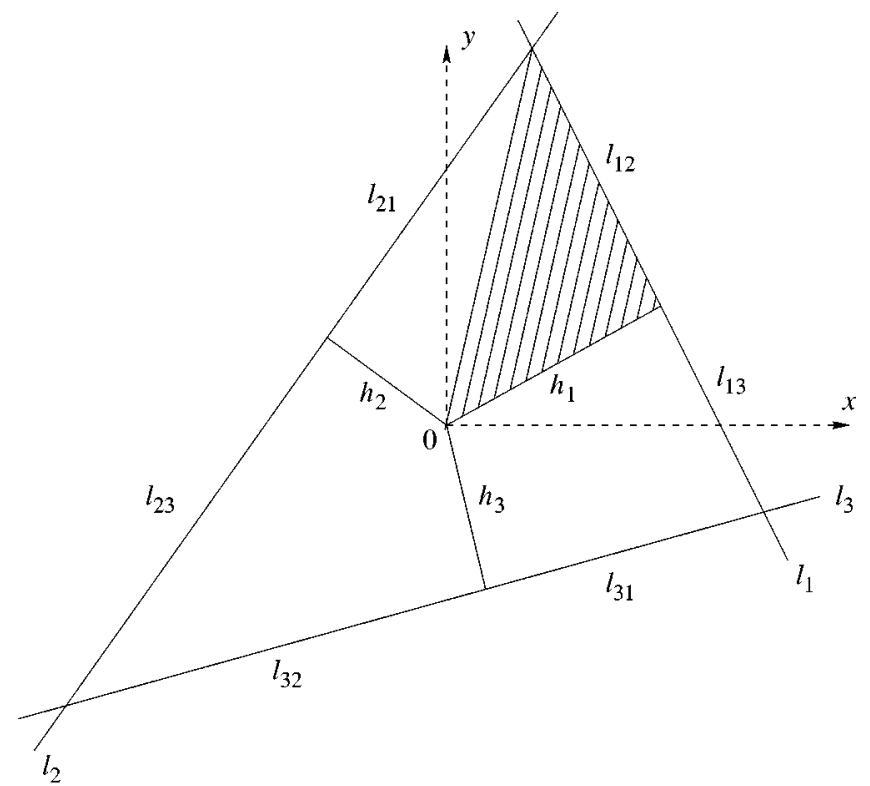

FIG. 5. The splitting of the region $R$ of integration into six nonoverlapping pieces.

Using rotational symmetry, Eq. (3.33) can be given more explicitly as

$$
\begin{aligned}
\bar{F}= & f\left(h_{1}, \ell_{12}, \bar{D}\right)+f\left(h_{2}, \ell_{23}, \bar{D}\right)+f\left(h_{3}, \ell_{31}, \bar{D}\right) \\
& +f\left(h_{1}, \ell_{13}, \bar{D}\right)+f\left(h_{2}, \ell_{21}, \bar{D}\right)+f\left(h_{3}, \ell_{32}, \bar{D}\right),
\end{aligned}
$$

where

$$
f(h, \ell, \bar{D})=\int d x d y\left(-x^{2}-y^{2}+\bar{D}\right)^{-3 / 2},
$$

with the region of integration shown in Fig. 6. The sign of this $f(h, \ell, \bar{D})$ is positive when

$$
h>0, \quad \ell>0, \quad \text { and } \quad \bar{D}>h^{2}+\ell^{2} \text {. }
$$

It has the property that

$$
\begin{aligned}
f(h, \ell, \bar{D}) & =-f(-h, \ell, \bar{D})=-f(h,-\ell, \bar{D}) \\
& =f(-h,-\ell, \bar{D}),
\end{aligned}
$$

which implies that it vanishes when $h=0$ and/or $\ell=0$, viz.,

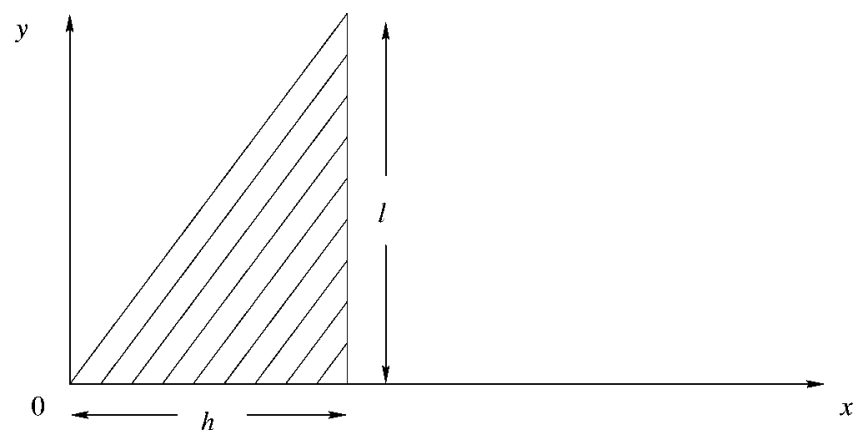

FIG. 6. The region of integration for the $f(h, l, \bar{D})$ of Eq. (3.35). 


$$
f(0, \ell, \bar{D})=f(h, 0, \bar{D})=0 .
$$

It remains to calculate this $f(h, \ell, \bar{D})$ explicitly. Using polar coordinates, this is straightforward and the result is

$$
f(h, l, \bar{D})=\frac{1}{\sqrt{\bar{D}}}\left[\arctan \left(\frac{l}{h} \sqrt{\frac{\bar{D}}{\bar{D}-h^{2}-l^{2}}}\right)-\arctan \frac{l}{h}\right],
$$

where both arctangents should be taken between $-\pi / 2$ and $\pi / 2$.

By Eqs. (3.26) and (3.34), this completes the evaluation of $F$ as defined by Eq. (3.1).

\section{F. Result}

The remaining task is to simplify the above answers in order to get a manageable expression for $F$ to be used in the following sections.

It is seen from Eq. (3.12) that the three $\theta$ 's are related to each other in a very simple way. Thus, the first step is to get an expression for these $\theta$ 's that exhibits explicitly these simple relations. This desired expression is

$$
e^{2 i \theta_{1}}=-\frac{1}{\sqrt{A-B}}\left(M_{1}^{2}+e^{-i 2 \pi / 3} M_{2}^{2}+e^{i 2 \pi / 3} M_{3}^{2}\right) .
$$

This implies simplification of the relation between the $\theta_{j}^{\prime}$ and $\theta_{j}$ as given by Eq. (3.31):

$$
\cos \theta_{1}^{\prime}=\frac{\sqrt{2 \lambda_{-}}}{M_{1}} \cos \theta_{1}, \quad \sin \theta_{1}^{\prime}=\frac{\sqrt{2 \lambda_{+}}}{M_{1}} \sin \theta_{1},
$$

$$
\begin{aligned}
& \cos \theta_{2}^{\prime}=\frac{\sqrt{2 \lambda_{-}}}{M_{2}} \cos \theta_{2}, \quad \sin \theta_{2}^{\prime}=\frac{\sqrt{2 \lambda_{+}}}{M_{2}} \sin \theta_{2}, \\
& \cos \theta_{3}^{\prime}=\frac{\sqrt{2 \lambda_{-}}}{M_{3}} \cos \theta_{3}, \quad \sin \theta_{3}^{\prime}=\frac{\sqrt{2 \lambda_{+}}}{M_{3}} \sin \theta_{3} .
\end{aligned}
$$

In particular, we have

$$
\begin{gathered}
\sin \left(\theta_{2}^{\prime}-\theta_{1}^{\prime}\right)=-\frac{\sqrt{-A+2 B}}{2 M_{1} M_{2}}, \\
\cos \left(\theta_{2}^{\prime}-\theta_{1}^{\prime}\right)=-\frac{M_{1}^{2}+M_{2}^{2}-M_{3}^{2}}{2 M_{1} M_{2}} .
\end{gathered}
$$

Note the minus signs.

In view of Eq. (3.18), the following nice relations are especially useful:

$$
\begin{aligned}
& \bar{D}-h_{1}^{2}-\ell_{12}^{2}=\bar{D}-h_{2}^{2}-\ell_{21}^{2}=m_{3}^{2}, \\
& \bar{D}-h_{2}^{2}-\ell_{23}^{2}=\bar{D}-h_{3}^{2}-\ell_{32}^{2}=m_{1}^{2}, \\
& \bar{D}-h_{3}^{2}-\ell_{31}^{2}=\bar{D}-h_{1}^{2}-\ell_{13}^{2}=m_{2}^{2} .
\end{aligned}
$$

Since the factor $-A+2 B$ appears very often, it is convenient to define, for $j=1,2,3$,

$$
\hat{\alpha}_{j}=(-A+2 B) \bar{\alpha}_{j} \quad \text { and } \quad \hat{D}=(-A+2 B) \bar{D} .
$$

From Eqs. (3.15) and (3.16), these quantities are given by

$$
\begin{gathered}
\hat{\alpha}_{1}=M_{1}^{2}\left(M_{2}^{2}+M_{3}^{2}-M_{1}^{2}\right)-M_{1}^{2}\left(m_{2}^{2}+m_{3}^{2}-2 m_{1}^{2}\right)-\left(M_{2}^{2}-M_{3}^{2}\right)\left(m_{2}^{2}-m_{3}^{2}\right), \\
\hat{\alpha}_{2}=M_{2}^{2}\left(M_{3}^{2}+M_{1}^{2}-M_{2}^{2}\right)-M_{2}^{2}\left(m_{3}^{2}+m_{1}^{2}-2 m_{2}^{2}\right)-\left(M_{3}^{2}-M_{1}^{2}\right)\left(m_{3}^{2}-m_{1}^{2}\right), \\
\hat{\alpha}_{3}=M_{3}^{2}\left(M_{1}^{2}+M_{2}^{2}-M_{3}^{2}\right)-M_{3}^{2}\left(m_{1}^{2}+m_{2}^{2}-2 m_{3}^{2}\right)-\left(M_{1}^{2}-M_{2}^{2}\right)\left(m_{1}^{2}-m_{2}^{2}\right), \\
\hat{D}=M_{1}^{2} M_{2}^{2} M_{3}^{2}+m_{1}^{2} M_{1}^{2}\left(-M_{1}^{2}+M_{2}^{2}+M_{3}^{2}\right)+m_{2}^{2} M_{2}^{2}\left(M_{1}^{2}-M_{2}^{2}+M_{3}^{2}\right)+m_{3}^{2} M_{3}^{2}\left(M_{1}^{2}+M_{2}^{2}-M_{3}^{2}\right)+M_{1}^{2}\left(m_{1}^{2}-m_{2}^{2}\right)\left(m_{1}^{2}-m_{3}^{2}\right) \\
+M_{2}^{2}\left(m_{2}^{2}-m_{3}^{2}\right)\left(m_{2}^{2}-m_{1}^{2}\right)+M_{3}^{2}\left(m_{3}^{2}-m_{1}^{2}\right)\left(m_{3}^{2}-m_{2}^{2}\right) .
\end{gathered}
$$

In terms of these quantities, the final result for $F$ is

$$
\begin{aligned}
F= & \frac{2}{\sqrt{\hat{D}}}\left\{\arctan \frac{\left(M_{1}^{2}+m_{2}^{2}-m_{3}^{2}\right) \sqrt{\hat{D}}}{\hat{\alpha}_{1} m_{3}}+\arctan \frac{\left(M_{1}^{2}-m_{2}^{2}+m_{3}^{2}\right) \sqrt{\hat{D}}}{\hat{\alpha}_{1} m_{2}}+\arctan \frac{\left(M_{2}^{2}+m_{3}^{2}-m_{1}^{2}\right) \sqrt{\hat{D}}}{\hat{\alpha}_{2} m_{1}}+\arctan \frac{\left(M_{2}^{2}-m_{3}^{2}+m_{1}^{2}\right) \sqrt{\hat{D}}}{\hat{\alpha}_{2} m_{3}}\right. \\
& \left.+\arctan \frac{\left(M_{3}^{2}+m_{1}^{2}-m_{2}^{2}\right) \sqrt{\hat{D}}}{\hat{\alpha}_{3} m_{2}}+\arctan \frac{\left(M_{3}^{2}-m_{1}^{2}+m_{2}^{2}\right) \sqrt{\hat{D}}}{\hat{\alpha}_{3} m_{1}}-\pi\right\} .
\end{aligned}
$$




\section{NONFORWARD SECOND-ORDER AMPLITUDE}

In this section, we shall check the result in Sec. III and the analytic continuation in $m_{3}$ by going to the limit corresponding to the special case of nonforward elastic scattering. We will also discuss the singularities in this case and introduce an approximation for $F$ which singles out the pole terms for small $\Delta$. In addition, we check the limit $\Delta \equiv M_{3} \rightarrow 0$ to obtain the forward result and compare it with the answer in paper I.

For nonforward elastic scattering, we have

$$
\begin{array}{ll}
M_{1}^{2}=M_{2}^{2}=k^{2}, & M_{3}^{2}=\Delta^{2}, \\
m_{1}^{2}=m_{2}^{2}=\mu^{2}, & m_{3}^{2}=m^{2} ;
\end{array}
$$

later, we want to continue in $m^{2}$ from $m^{2}>0$ to

$$
m^{2}=-\left(k^{2}+a^{2}\right)-i \epsilon, \quad m=-i \sqrt{k^{2}+a^{2}} .
$$

The minus sign before the square root is crucial to get the correct result. In the present case, the $A$ and $B$ defined by Eq. (3.8) are

$$
A=2 k^{4}+\Delta^{4}
$$

and

$$
B=k^{4}+2 \Delta^{2} k^{2} \text {. }
$$

We take $\Delta^{2}<k^{2}$, and hence

$$
\sqrt{-A+2 B}=\Delta \sqrt{4 k^{2}-\Delta^{2}}
$$

From Eqs. (3.29), (3.12), and (3.16), after some algebra one gets (note that we now have $\theta_{1}=\pi / 3, \theta_{2}=-\pi / 3$, and $\left.\theta_{3}=\pi\right)$

$$
h_{1}=h_{2}=\frac{\Delta}{2 k} \frac{k^{2}+\mu^{2}-m^{2}}{\sqrt{4 k^{2}-\Delta^{2}}}, \quad h_{3}=\frac{k^{2}-\Delta^{2} / 2-\mu^{2}+m^{2}}{\sqrt{4 k^{2}-\Delta^{2}}} \text {. }
$$

The $\ell_{i j}$ 's are defined in Eq. (3.30). For the present case, it follows from Eq. (3.31) that

$$
\theta_{1}^{\prime}=\frac{\pi}{2}-\sin ^{-1} \frac{\Delta}{2 k}, \quad \theta_{2}^{\prime}=-\theta_{1}^{\prime}, \quad \theta_{3}^{\prime}=\pi
$$

Hence we obtain

$$
\begin{gathered}
\ell_{12}=\ell_{21}=\frac{k^{2}+\mu^{2}-m^{2}}{2 k}, \\
\ell_{32}=\ell_{31}=\frac{\Delta}{2}, \\
\ell_{13}=\ell_{23}=\frac{k^{2}-\mu^{2}+m^{2}}{2 k} .
\end{gathered}
$$

From Eq. (3.34) we can obtain $\bar{F}$, which is defined by Eq. (3.26),

$$
\begin{aligned}
\bar{F}= & 2\left[f\left(\frac{\Delta}{2 k} \frac{k^{2}+\mu^{2}-m^{2}}{\sqrt{4 k^{2}-\Delta^{2}}}, \frac{k^{2}+\mu^{2}-m^{2}}{2 k}, \bar{D}\right)\right. \\
& +f\left(\frac{\Delta}{2 k} \frac{k^{2}+\mu^{2}-m^{2}}{\sqrt{4 k^{2}-\Delta^{2}}}, \frac{k^{2}-\mu^{2}+m^{2}}{2 k}, \bar{D}\right) \\
& \left.+f\left(\frac{k^{2}-\Delta^{2} / 2-\mu^{2}+m^{2}}{\sqrt{4 k^{2}-\Delta^{2}}}, \frac{\Delta}{2}, \bar{D}\right)\right] .
\end{aligned}
$$

From Eq. (3.15) we get

$$
\bar{D}=\frac{k^{4}+2 \mu^{2} k^{2}+m^{2}\left(2 k^{2}-\Delta^{2}\right)+\left(\mu^{2}-m^{2}\right)^{2}}{4 k^{2}-\Delta^{2}} .
$$

The integral $f(h, \ell, \bar{D})$ is given in Eq. (3.38), and can be written in two equivalent ways:

$$
\begin{aligned}
f(h, \ell, \bar{D}) & =\frac{1}{\sqrt{\bar{D}}}\left\{\arctan \left(\frac{\ell}{h} \sqrt{\frac{\bar{D}}{\bar{D}-h^{2}-\ell^{2}}}\right)-\arctan \frac{\ell}{h}\right\} \\
& =\frac{1}{\sqrt{\bar{D}}}\left\{\arctan \frac{h}{\ell}-\arctan \left(\frac{h}{\ell} \sqrt{\frac{\bar{D}-h^{2}-\ell^{2}}{\bar{D}}}\right)\right\} .
\end{aligned}
$$

For the first two $f$ 's in Eq. (4.9) we use the second form of $f$ given in Eq. (4.11), and for the last $f$ we use the first line of Eq. (4.11). After some algebra, and making use of the simplifying relations (3.42), we obtain

$$
\begin{aligned}
\bar{F}= & \frac{2}{\sqrt{\bar{D}}}\left\{-\arctan \left(\frac{\Delta m}{\sqrt{4 k^{2}-\Delta^{2}} \sqrt{\bar{D}}}\right)\right. \\
& -\arctan \left(\frac{\Delta}{\sqrt{4 k^{2}-\Delta^{2}}} \frac{k^{2}+\mu^{2}-m^{2}}{k^{2}-\mu^{2}+m^{2}} \frac{\mu}{\sqrt{\bar{D}}}\right) \\
& \left.+\arctan \left(\frac{\Delta \sqrt{4 k^{2}-\Delta^{2}} \sqrt{\bar{D}}}{2\left(k^{2}-\mu^{2}+m^{2}-\Delta^{2} / 2\right) \mu}\right)\right\} .
\end{aligned}
$$

It is crucial for the validity of Eq. (4.12) to note that

$$
\tan ^{-1} \frac{h_{1}}{\ell_{12}}+\tan ^{-1} \frac{h_{2}}{\ell_{13}}-\tan ^{-1} \frac{\ell_{32}}{h_{3}}=0,
$$

with $h_{j}$ and $\ell_{i j}$ given by Eqs. (4.6) and (4.8). In terms of the $\hat{D}$ of Eq. (3.44), which reduces to

$$
\hat{D}=\Delta^{2}\left[k^{4}+2 \mu^{2} k^{2}+m^{2}\left(2 k^{2}-\Delta^{2}\right)+\left(\mu^{2}-m^{2}\right)^{2}\right]
$$

for the present case, the desired $F$ is given by 


$$
\begin{aligned}
F= & \frac{4}{\sqrt{\hat{D}}}\left\{-\arctan \frac{m \Delta^{2}}{\sqrt{\hat{D}}}-\arctan \frac{\left(k^{2}+\mu^{2}-m^{2}\right) \mu \Delta^{2}}{\left(k^{2}-\mu^{2}+m^{2}\right) \sqrt{\hat{D}}}\right. \\
& \left.+\arctan \frac{\sqrt{\hat{D}}}{2\left(k^{2}-\mu^{2}+m^{2}-\Delta^{2} / 2\right) \mu}\right\} .
\end{aligned}
$$

Since the region of small $\Delta$ is of central importance, it is convenient to remove the factor of $\Delta^{2}$ from $\hat{D}$. Therefore, define in general

$$
\widetilde{D}=\frac{\hat{D}}{M_{3}^{2}},
$$

which is $\hat{D} / \Delta^{2}$ for the present case. This leads immediately to

$$
\begin{aligned}
F= & \frac{4}{\Delta \sqrt{\widetilde{D}}}\left\{-\arctan \frac{\Delta m}{\sqrt{\widetilde{D}}}-\arctan \frac{\mu \Delta}{\sqrt{\widetilde{D}}} \frac{k^{2}+\mu^{2}-m^{2}}{k^{2}-\mu^{2}+m^{2}}\right. \\
& \left.+\arctan \frac{\Delta \sqrt{\widetilde{D}}}{2 \mu\left(k^{2}-\mu^{2}+m^{2}-\Delta^{2} / 2\right)}\right\} .
\end{aligned}
$$

The analytic continuation of $F$ can be accomplished by setting $m^{2}=-k^{2}-a^{2}-i \epsilon$ in Eq. (4.17) and using Eq. (4.2), resulting in

$$
\begin{aligned}
F= & \frac{4}{\Delta \sqrt{\widetilde{D}}}\left\{\arctan \frac{+i \sqrt{k^{2}+a^{2}} \Delta}{\sqrt{\widetilde{D}}}\right. \\
& +\arctan \frac{\mu \Delta\left(2 k^{2}+\mu^{2}+a^{2}\right)}{\sqrt{\widetilde{D}}\left(a^{2}+\mu^{2}\right)} \\
& \left.-\arctan \frac{\Delta \sqrt{\widetilde{D}}}{2 \mu\left(\mu^{2}+a^{2}+\Delta^{2} / 2\right)}\right\},
\end{aligned}
$$

where now $\widetilde{D}$ is

$$
\widetilde{D}=\left(\mu^{2}+a^{2}\right)^{2}+4 k^{2} \mu^{2}+\left(k^{2}+a^{2}\right) \Delta^{2} .
$$

One can make two checks on Eq. (4.18). First for the forward cases [3], $\Delta \rightarrow 0$, we get

$$
\left.F\right|_{\Delta \rightarrow 0}=\frac{2}{\mu\left[\left(\mu^{2}+a^{2}\right)^{2}+4 k^{2} \mu^{2}\right]}\left\{2 \mu i \sqrt{k^{2}+a^{2}}-\left(a^{2}-\mu^{2}\right)\right\} .
$$

After multiplication by an overall factor of $\pi^{2} / 2$, this agrees with the forward result in Ref. [3], and also with Eq. (4.7) of paper I. Second, for the nonforward case, $\Delta \neq 0$, we can carry out the non-Euclidean calculation of $F$ directly using methods analogous to those used in Ref. [3]. In other words, one can evaluate the integral for $\widetilde{F}(\Delta)$ given in Eq. (2.6) with $\vec{k}^{2}=(\vec{k}+\vec{\Delta})^{2}$. Using contour integration, the result for this integral is identical with Eq. (4.17), again confirming our analytic continuation algorithm.
Next we discuss the singularities of $F$ given by Eq. (4.18). From the zeros of $\widetilde{D}$ we get singularities at

$$
k= \pm i \sqrt{\left(\mu^{2}+a^{2}\right)^{2}+a^{2} \Delta^{2} 4 \mu^{2}+\Delta^{2}} .
$$

From the $\arctan z$ when $z= \pm i$, we get, from the first term in Eq. (4.18),

$$
\frac{i \sqrt{k^{2}+a^{2}} \Delta}{\sqrt{\widetilde{D}}}= \pm i
$$

which leads to

$$
k= \pm i\left(\frac{\mu^{2}+a^{2}}{2 \mu}\right) .
$$

This is the position of the original singularity of the forward case. The other two arctangents in Eq. (4.18) do not lead to any singularity that approaches $k=i\left(a^{2}+\mu^{2}\right) / 2 \mu$ as $\vec{\Delta} \rightarrow 0$, while the singularities in Eq. (4.21) do.

Up to this point everything is exact. We now want to introduce approximations in order to study the behavior of $F$ near the forward direction, i.e., $\Delta$ small, in the vicinity of the singularity at $k^{2}=-\left(a^{2}+\mu^{2}\right)^{2} / 4 \mu^{2}$ or, more precisely, at $k=+i\left(a^{2}+\mu^{2}\right) / 2 \mu$ [see the discussion after Eq. (4.8) of paper I].

We take $a>\mu>0$. Also we choose $a, \mu$, and $(a-\mu)$ to be all of the same order of magnitude; i.e., we take $a$ not too close to $\mu$.

We concentrate on the region where $\Delta$ is small, i.e.,

$$
\Delta^{2} \ll \mu^{2} .
$$

Next, we define a new variable $\alpha$ by

$$
k^{2}=-\left(\frac{a^{2}+\mu^{2}}{2 \mu}\right)^{2}+\alpha \frac{\Delta^{2}}{4} .
$$

Here $\alpha$ is taken to be $O(1)$, but sometimes we will take large $\alpha$ to recover the forward result. In some sense $\alpha$ measures the distance from the pole in units of $\Delta^{2} / 4$, and $\alpha$ is dimensionless. We thus have

$$
k=+i\left[\frac{a^{2}+\mu^{2}}{2 \mu}-\frac{\alpha \Delta^{2}}{4} \frac{\mu}{a^{2}+\mu^{2}}\right]+O\left(\Delta^{4}\right)
$$

and

$$
i m=\sqrt{k^{2}+a^{2}}=i\left[\frac{a^{2}-\mu^{2}}{2 \mu}-\frac{\alpha \Delta^{2}}{4} \frac{\mu}{a^{2}-\mu^{2}}\right]+O\left(\Delta^{4}\right) .
$$

From Eq. (4.19) we get

$$
\sqrt{\widetilde{D}}=\frac{\Delta}{2 \mu} \sqrt{4 \alpha \mu^{4}-\left(a^{2}-\mu^{2}\right)^{2}}+O\left(\Delta^{3}\right) .
$$

Going back to Eq. (4.18), we obtain 


$$
\begin{aligned}
F= & \frac{16 \mu}{\Delta^{2} \sqrt{4 \alpha \mu^{4}-\left(a^{2}-\mu^{2}\right)^{2}}} \\
& \times\left\{\arctan \frac{-\left(a^{2}-\mu^{2}\right)}{\sqrt{4 \alpha \mu^{4}-\left(a^{2}-\mu^{2}\right)^{2}}}+O\left(\Delta^{2}\right)\right\} .
\end{aligned}
$$

Note that the third arctan in Eq. (4.18) is $O\left(\Delta^{2}\right)$ and hence is neglected.

Finally, since we are interested in small $\alpha$, we rewrite Eq. (4.29) as

$$
\begin{aligned}
F= & \frac{16 \mu}{\Delta^{2} \sqrt{\left(a^{2}-\mu^{2}\right)^{2}-4 \alpha \mu^{4}}} \\
& \times\left\{\operatorname{arctanh}\left(\frac{a^{2}-\mu^{2}}{\sqrt{\left(a^{2}-\mu^{2}\right)^{2}-4 \alpha \mu^{4}}}\right)+O\left(\Delta^{2}\right)\right\} .
\end{aligned}
$$

Here, arctanh is taken such that $F \rightarrow O\left(\alpha^{-1}\right)$ as $\alpha \rightarrow \infty$.

\section{FOURTH ORDER}

The second-order $\widetilde{F}$ that appears in the expression (2.5) for $F^{(4)}$ is both nonforward and nonelastic, $k^{2} \neq k^{\prime 2}$. It corresponds to the following choice for the $M_{j}$ 's and the $m_{j}$ 's:

$$
\begin{gathered}
M_{1}^{2}=k^{2}, \quad M_{2}^{2}=k^{\prime 2}, \quad M_{3}^{2}=\Delta^{2}, \\
m_{1}^{2}=m_{2}^{2}=\mu^{2}, \quad m_{3}^{2}=m^{2}=-\left(k^{2}+a^{2}\right)-i \epsilon .
\end{gathered}
$$

From Eq. (3.8), we get, for this case,

$$
-A+2 B=-\left(k^{2}-k^{\prime 2}\right)^{2}+2\left(k^{2}+k^{\prime 2}\right) \Delta^{2}-\Delta^{4} .
$$

From Eqs. (3.44) and (4.16), we have, in this case,

$$
\begin{aligned}
\Delta^{2} \widetilde{D}= & -\mu^{2}\left(k^{2}-k^{\prime 2}\right)^{2}+\left[k^{2} k^{\prime 2}+\left(m^{2}+\mu^{2}\right)\left(k^{2}+k^{\prime 2}\right)\right. \\
& \left.+\left(m^{2}-\mu^{2}\right)^{2}\right] \Delta^{2}-m^{2} \Delta^{4} .
\end{aligned}
$$

In order to maintain the symmetry, we define a $\kappa$ and a $\delta$ as follows:

$$
\kappa^{2} \equiv \frac{1}{2}\left(k^{2}+k^{\prime 2}\right), \quad \Delta^{2} \delta \equiv k^{2}-k^{\prime 2}
$$

Hence,

$$
k^{2}=\kappa^{2}+\frac{1}{2}\left(\delta \Delta^{2}\right) \quad \text { and } \quad k^{\prime 2}=\kappa^{2}-\frac{1}{2}\left(\delta \Delta^{2}\right) .
$$

The elastic case studied in the previous section corresponds to $\delta=0$ and $\kappa=k=k^{\prime}$. To keep the symmetry, we write

$$
m^{2} \equiv-\kappa^{2}-\mathcal{A}^{2}
$$

where

$$
\mathcal{A}^{2}=a^{2}-\kappa^{2}+k^{2}=a^{2}+\frac{1}{2} \delta \Delta^{2} .
$$

In this notation we have
$\widetilde{D}=\kappa^{2}\left(4 \mu^{2}+\Delta^{2}\right)+\left(\mathcal{A}^{2}+\mu^{2}\right)^{2}+\mathcal{A}^{2} \Delta^{2}-\frac{1}{4} \delta^{2} \Delta^{4}-\mu^{2} \delta^{2} \Delta^{2}$

and

$$
(-A+2 B)=\Delta^{2}\left[4 \kappa^{2}-\left(1+\delta^{2}\right) \Delta^{2}\right] .
$$

We now carry out an approximation for $\widetilde{F}$ near $\kappa=+i\left(a^{2}+\mu^{2}\right) / 2 \mu$ and for $\Delta$ small, similar to the one carried out in the previous section. The singularities near $\kappa=+i\left(a^{2}+\mu^{2}\right) / 2 \mu$ are at

$$
k=+i \frac{a^{2}+\mu^{2}}{2 \mu}, \quad k^{\prime}=+i \frac{a^{2}+\mu^{2}+\delta^{2} \Delta^{2}}{2 \mu} .
$$

To keep the symmetry, it is convenient to use $\mathcal{A}$ instead of $a$. Next we define $\alpha$ and $\alpha^{\prime}$ by

$$
k^{2}=-\left(\frac{\mathcal{A}^{2}+\mu^{2}}{2 \mu}\right)^{2}+\frac{\alpha \Delta^{2}}{4}, \quad k^{\prime 2}=-\left(\frac{\mathcal{A}^{2}+\mu^{2}}{2 \mu}\right)^{2}+\frac{\alpha^{\prime} \Delta^{2}}{4} .
$$

Writing

$$
\bar{\alpha} \equiv \frac{1}{2}\left(\alpha+\alpha^{\prime}\right),
$$

we then obtain

$$
\kappa^{2}=-\left(\frac{\mathcal{A}^{2}+\mu^{2}}{2 \mu}\right)^{2}+\frac{\bar{\alpha} \Delta^{2}}{4}
$$

and

$$
\delta=\frac{\alpha-\alpha^{\prime}}{4} .
$$

To lowest order in $\Delta$ we have

$$
\begin{array}{r}
k=i\left[\frac{\mathcal{A}^{2}+\mu^{2}}{2 \mu}-\frac{\alpha \Delta^{2}}{4} \frac{\mu}{\mathcal{A}^{2}+\mu^{2}}+O\left(\Delta^{4}\right)\right], \\
k^{\prime}=i\left[\frac{\mathcal{A}^{2}+\mu^{2}}{2 \mu}-\frac{\alpha^{\prime} \Delta^{2}}{4} \frac{\mu}{\mathcal{A}^{2}+\mu^{2}}+O\left(\Delta^{4}\right)\right], \\
\kappa=i\left[\frac{\mathcal{A}^{2}+\mu^{2}}{2 \mu}-\frac{\bar{\alpha} \Delta^{2}}{4} \frac{\mu}{\mathcal{A}^{2}+\mu^{2}}+O\left(\Delta^{4}\right)\right],
\end{array}
$$

and

$$
m=-i \sqrt{\kappa^{2}+\mathcal{A}^{2}}=\frac{\mathcal{A}^{2}-\mu^{2}}{2 \mu}-\frac{\bar{\alpha} \Delta^{2}}{4} \frac{\mu^{2}}{\mathcal{A}^{2}-\mu^{2}}+O\left(\Delta^{4}\right) .
$$

The approximate form for $\widetilde{D}$ is now given by

$$
\widetilde{D}=\frac{\Delta^{2}}{4 \mu^{2}}\left[4 \mu^{4} \bar{\alpha}-\left(\mathcal{A}^{2}-\mu^{2}\right)^{2}-4 \mu^{4} \delta^{2}+O\left(\Delta^{2}\right)\right] .
$$


With our new variables it is now easy to see that to obtain $\widetilde{F}$ in this case we can follow the previous section with the substitutions

$$
k \rightarrow \kappa, \quad a \rightarrow \mathcal{A}, \quad \alpha \rightarrow \bar{\alpha}
$$

The only quantities that are modified are $\widetilde{D}, h_{1}$, and $h_{2}=\left.h_{1}\right|_{\delta \rightarrow-\delta}$. In fact, for $h_{1}$ and $h_{2}$ we get, neglecting higher orders in $\Delta$,

$$
\begin{aligned}
& h_{1}=\frac{\Delta}{4 \kappa^{2}}\left[\left(2 \kappa^{2}+\mu^{2}+\mathcal{A}^{2}\right)+\left(\mu^{2}+\mathcal{A}^{2}\right) \delta\right], \\
& h_{2}=\frac{\Delta}{4 \kappa^{2}}\left[\left(2 \kappa^{2}+\mu^{2}+\mathcal{A}^{2}\right)-\left(\mu^{2}+\mathcal{A}^{2}\right) \delta\right] .
\end{aligned}
$$

After some algebra we get, from Eq. (3.34),

$$
\begin{aligned}
F= & \frac{8 \mu}{\Delta^{2} \sqrt{\left(\mathcal{A}^{2}-\mu^{2}\right)^{2}-4\left(\bar{\alpha}-\delta^{2}\right) \mu^{4}}} \\
& \times\left\{\operatorname{arctanh}\left[\frac{\mathcal{A}^{2}-\mu^{2}(1+2 \delta)}{\sqrt{\left(\mathcal{A}^{2}-\mu^{2}\right)^{2}-4\left(\bar{\alpha}-\delta^{2}\right) \mu^{4}}}\right]\right. \\
& \left.+\operatorname{arctanh}\left[\frac{\mathcal{A}^{2}-\mu^{2}(1-2 \delta)}{\sqrt{\left(\mathcal{A}^{2}-\mu^{2}\right)^{2}-4\left(\bar{\alpha}-\delta^{2}\right) \mu^{4}}}\right]+O\left(\Delta^{2}\right)\right\} .
\end{aligned}
$$

Note that, as $\delta \rightarrow 0$, Eq. (5.20) becomes identical with Eq. (4.29) with $\bar{\alpha} \rightarrow \alpha$ and $\mathcal{A} \rightarrow a$. In fact, to be consistent, one should replace $\mathcal{A}$ by $a$ in Eq. (5.20), since from Eq. (5.7) we have $\mathcal{A}^{2}=a^{2}+O\left(\Delta^{2}\right)$. The final result for $F$ is

$$
\begin{aligned}
F \cong & \frac{8 \mu}{\Delta^{2} \sqrt{\left(a^{2}-\mu^{2}\right)^{2}-4\left(\bar{\alpha}-\delta^{2}\right) \mu^{4}}} \\
& \times\left[\operatorname{arctanh} \frac{a^{2}-\mu^{2}(1+2 \delta)}{\sqrt{\left(a^{2}-\mu^{2}\right)^{2}-4\left(\bar{\alpha}-\delta^{2}\right) \mu^{4}}}\right. \\
& \left.+\operatorname{arctanh} \frac{a^{2}-\mu^{2}(1-2 \delta)}{\sqrt{\left(a^{2}-\mu^{2}\right)^{2}-4\left(\bar{\alpha}-\delta^{2}\right) \mu^{4}}}\right] .
\end{aligned}
$$

We recall that $a>\mu>0$ throughout. This becomes exact as $\Delta \rightarrow 0$.

In the rest of this section, we shall use this approximation for $F$ in Eq. (2.5) to determine the behavior of the fourthorder amplitude near the point

$$
k=i\left(\frac{a^{2}+\mu^{2}}{2 \mu}\right),
$$

remembering that

$$
\widetilde{F}=\frac{\pi^{2}}{2} F
$$

On the right-hand side of Eq. (2.5), $\vec{k}$ and $\vec{k}^{\prime}=\vec{k}+\vec{\Delta}$ play different roles: $k^{2}$ is fixed while $k^{\prime 2}$ is integrated over. It is convenient to use as integration variables $\Delta^{2}$ and $\delta$, instead of $\vec{\Delta}$. From $k^{\prime 2}=(\vec{k}+\vec{\Delta})^{2}$ and Eq. (5.4), we get

$$
\vec{k} \cdot \vec{\Delta}=-\frac{1}{2 k}(1+\delta) \Delta^{2}
$$

The volume integration is

$$
\int d^{3} \Delta=-\frac{\pi}{2 k} \int_{-\infty}^{+\infty} d \delta \int_{0}^{\infty} \Delta^{2} d \Delta^{2} .
$$

Hence,

$$
F^{(4)}=-\frac{\pi}{2 k} \int_{-\infty}^{+\infty} \frac{d \delta}{-\delta-i \epsilon} \int_{0}^{\infty} d \Delta^{2}\left[\frac{\pi^{2}}{2} F(\Delta)\right]^{2} .
$$

Using the approximation (5.21) for $F$, and setting $k=i\left(a^{2}+\mu^{2}\right) / 2 \mu$, we obtain

$$
\begin{aligned}
F^{(4)} \cong & \frac{\pi^{5} i \mu}{4\left(a^{2}+\mu^{2}\right)} \int_{-\infty}^{+\infty} \frac{d \delta}{-\delta-i \epsilon} \\
& \times \int_{0}^{\infty} d \Delta^{2}\left\{\frac{8 \mu}{\Delta^{2} \sqrt{\left(a^{2}-\mu^{2}\right)^{2}-4\left(\bar{\alpha}-\delta^{2}\right) \mu^{4}}}\right. \\
& \times\left[\operatorname{arctanh} \frac{a^{2}-\mu^{2}(1+2 \delta)}{\sqrt{\left(a^{2}-\mu^{2}\right)^{2}-4\left(\bar{\alpha}-\delta^{2}\right) \mu^{4}}}\right. \\
& \left.\left.+\operatorname{arctanh} \frac{a^{2}-\mu^{2}(1-2 \delta)}{\sqrt{\left(a^{2}-\mu^{2}\right)^{2}-4\left(\bar{\alpha}-\delta^{2}\right) \mu^{4}}}\right]\right\}^{2} .
\end{aligned}
$$

We now have to write out an expression for $\bar{\alpha}$ in terms of the integration variables. From Eqs. (5.12) and (5.14), we get

$$
\bar{\alpha}=\alpha-2 \delta \text {. }
$$

However, from Eqs. (5.7), (5.10), and (5.11), we have the definition of $\alpha$ in terms of $\mathcal{A}, \mu, \Delta^{2}$, and $k^{2}$. We now define $\epsilon$ as

$$
\epsilon=k^{2}-\left[-\left(\frac{a^{2}+\mu^{2}}{2 \mu}\right)^{2}\right],
$$

so that

$$
\epsilon=\frac{\Delta^{2}}{4}\left[\alpha-\left(\frac{a^{2}+\mu^{2}}{\mu^{2}}\right) \delta\right]+O\left(\Delta^{4}\right) .
$$

Hence, we have

$$
\bar{\alpha}=\frac{4 \epsilon}{\Delta^{2}}+\left(\frac{a^{2}+\mu^{2}}{\mu^{2}}\right) \delta-2 \delta=\frac{4 \epsilon}{\Delta^{2}}+\left(\frac{a^{2}-\mu^{2}}{\mu^{2}}\right) \delta .
$$

We define a new variable $x$ as 


$$
x \equiv \frac{4 \epsilon}{\Delta^{2}},
$$

and obtain

$$
\left(a^{2}-\mu^{2}\right)^{2}-4 \mu^{4}\left(\bar{\alpha}-\delta^{2}\right)=\left[\left(\frac{a^{2}-\mu^{2}}{2 \mu^{2}}-\delta\right)^{2}-x\right] 4 \mu^{4} \text {. }
$$

Setting

$$
v \equiv \frac{a^{2}-\mu^{2}}{2 \mu^{2}}, \quad v>0
$$

we finally get

$$
\begin{aligned}
F^{(4)}= & \left(\frac{1}{\epsilon}\right) \frac{-\pi^{5} i}{\mu\left(a^{2}+\mu^{2}\right)} \int_{-\infty}^{+\infty} \frac{d \delta}{\delta+i \epsilon} \int_{0}^{\infty} d x \\
& \times\left\{\frac { 1 } { \sqrt { ( v - \delta ) ^ { 2 } - x } } \left[\operatorname{arctanh} \frac{v-\delta}{\sqrt{(v-\delta)^{2}-x}}\right.\right. \\
& \left.\left.+\operatorname{arctanh} \frac{v+\delta}{\sqrt{(v-\delta)^{2}-x}}\right]\right\}^{2} .
\end{aligned}
$$

At this stage we have three possibilities. The singularity of $F_{0}^{(4)}$ at $k=+i\left(a^{2}+\mu^{2}\right) / 2 \mu$ is (i) stronger than a pole if the double integral in Eq. (5.35) is divergent, (ii) as strong as a pole if the double integral is convergent and nonzero, and (iii) weaker than a pole if the double integral is zero.

We proceed to show that (ii) is the correct option. In order to do this we have to be careful which sheet we are on, and introduce the continuation properly. We have already checked in several ways that we have to replace $a^{2}$ by $a^{2}+i \epsilon$ to get the proper continuation in $m_{3}$. Since $v=\left(a^{2}-\mu^{2}\right) / 2 \mu^{2}$, we then set

$$
v \rightarrow v+i \epsilon
$$

and Eq. (5.35) becomes

$$
F^{(4)}=\left(\frac{1}{\epsilon}\right) \frac{-\pi^{5} i}{2 \mu^{3}(v+1)} \int_{-\infty}^{+\infty} \frac{d \delta}{\delta+i \epsilon} \int_{0}^{\infty} d x I(\delta, x),
$$

where

$$
\begin{aligned}
I(\delta, x)= & \frac{1}{(v+i \epsilon-\delta)^{2}-x}\left\{\operatorname{arctanh} \frac{v+i \epsilon-\delta}{\sqrt{(v+i \epsilon-\delta)^{2}-x}}\right. \\
& \left.+\operatorname{arctanh} \frac{v+i \epsilon+\delta}{\sqrt{(v+i \epsilon-\delta)^{2}-x}}\right\}^{2} .
\end{aligned}
$$

Here $I$ is defined such that both arctanh terms $\rightarrow 0$ as $x \rightarrow+\infty$. Let $\xi$ be the quantity in the denominators:

$$
\begin{aligned}
\xi & =(v+i \epsilon-\delta)^{2}-x, \\
& =\left(v^{2}+\delta^{2}-x\right)+2 i \epsilon(v-\delta) .
\end{aligned}
$$

Thus, $\operatorname{Im} \xi>0$ for $\delta<v$ and $\operatorname{Im} \xi<0$ for $\delta>v$. The zeros of $\xi$ are given by

$$
\delta=v \pm \sqrt{x}+i \epsilon
$$

The $\operatorname{arctanh} z$ has branch points at $z= \pm 1$. Thus the first arctanh in Eq. (5.38), with $x>0$, has only a branch point at $\delta=\infty$, and no branch point in the finite $\delta$ plane. However, the second arctanh gives us a singularity in the $\delta$ plane located by

$$
(v+i \epsilon+\delta)^{2}=(v+i \epsilon-\delta)^{2}-x .
$$

This gives a branch point at

$$
\delta=-\frac{x}{4 v}+\frac{i \epsilon x}{4 v^{2}}, \quad x>0, v>0
$$

As $x$ increases from zero to $+\infty$, this branch point remains in the second quadrant.

Finally, $I(\delta, x)$ in Eq. (5.38) can be simplified by combining the two arctangents, and one gets

$$
I(\delta, x)=\left[\frac{1}{\sqrt{\xi}} \operatorname{arctanh} \frac{2 v \sqrt{\xi}}{(v+i \epsilon)^{2}-2(v+i \epsilon) \delta-x}\right]^{2},
$$

with $\xi$ given in Eq. (5.39). Note that replacing $\sqrt{\xi} \rightarrow-\sqrt{\xi}$ does not affect $I$, and there is no singularity in $I$ due to the point $\xi=0$.

The only branch point in $\delta$, for $x>0$, is given by Eq. (5.42), and lies in the second quadrant of the $\delta$ plane. This allows us to perform a Wick rotation for the $\delta$ integration in Eq. (5.37). Setting $\delta=-i y$, we obtain

$$
F^{(4)}=\left(\frac{1}{\epsilon}\right) \frac{-\pi^{5} i}{2 \mu^{3}(v+1)} \int_{-\infty}^{+\infty} \frac{d y}{y} \int_{0}^{\infty} d x J(y, x),
$$

where

$$
J(y, x)=I(\delta=-i y, x) .
$$

The contour for the $y$ integration circles above the pole at $y=0$. At this stage we can safely drop all $i \epsilon$ 's, and write

$$
J(y, x)=\frac{1}{(v-i y)^{2}-x}\left\{\operatorname{arctanh} \frac{2 v \sqrt{(v-i y)^{2}-x}}{v^{2}-2 i v y-x}\right\}^{2} .
$$

It is now clear that $F^{(4)}=\left(\epsilon^{-1}\right) \times$ finite number. The arctanh term vanishes as $x \rightarrow \infty$, and we know that $|\operatorname{arctanh} z|<C|z|$. This result eliminates option (i) listed below Eq. (5.35).

It remains to distinguish between the possibilities (ii) and (iii). The question is, is the double integral in Eq. (5.37) equal to zero for a range of values of $v, v=\left(a^{2}-\mu^{2}\right) / 2 \mu^{2}$ ?

We consider the case $v$ positive and small. This gives, from Eq. (5.43), 


$$
I(\delta, x)=\frac{4(v+i \epsilon)^{2}}{\left[(v+i \epsilon)^{2}-2(v+i \epsilon) \delta-x\right]^{2}}+O\left(v^{4}\right) .
$$

Substituting this in Eq. (5.37) and integrating over $x$ gives

$$
F^{(4)} \cong\left(\frac{1}{\epsilon}\right) \frac{\pi^{5} i}{2 \mu^{3}} \int_{-\infty}^{+\infty} \frac{d \delta}{\delta+i \epsilon}\left(\frac{4(v+i \epsilon)}{(v+i \epsilon)-2 \delta}\right)=\left(\frac{1}{\epsilon}\right) \pi^{5} i \frac{4 \pi i}{\mu^{3}}
$$

Hence, for small $v, F^{(4)}$ near the $k=i\left[\left(a^{2}+\mu^{2}\right) / 2 \mu\right]$ singularity behaves like $\left(\epsilon^{-1}\right) \times\left(-4 \pi^{6} / \mu^{3}\right)$. Thus we have eliminated option (iii).

We have shown that $F^{(4)}$ has a pole at $k=i\left[\left(a^{2}+\mu^{2}\right) / 2 \mu\right]$, but we have not actually excluded the possibility that there is also a branch cut at this point.

\section{DISCUSSION AND CONCLUSIONS}

It is shown in this paper that the fourth-order forward scattering amplitude $T_{22}^{(4)}(k)$, defined by Eqs. (2.2) and (2.3) for the present two-channel problem, has a singularity located at the same position as the corresponding second-order amplitude $T_{22}^{(2)}(k)$. The location of this singularity is on the physical sheet at

$$
k=+i \frac{a^{2}+\mu^{2}}{2 \mu}
$$

For $T_{22}^{(2)}(k)$, this singularity is a pole; for $T_{22}^{(4)}(k)$, this singularity has the strength of a pole. What this means is that the limit

$$
\lim _{k \rightarrow i\left(a^{2}+\mu^{2}\right) / 2 \mu}\left[k-i \frac{a^{2}+\mu^{2}}{2 \mu}\right] T_{22}^{(4)}(k)
$$

exists and is nonzero.

The major unsolved problems are the following.

(1) Is the singularity of $T_{22}^{(4)}(k)$ at the position (6.1) a pole or not? What needs to be decided is whether this singularity is isolated or not. If it is, then it has to be a pole. If not, then it is likely to be a branch cut. In imprecise language, we may say this is "the superposition of a pole and a branch cut." Efforts to answer this question have so far not been successful.

(2) Do higher-order forward scattering amplitudes $T_{22}^{(2 n)}(k)$, with $n>2$, for the present two-channel problem also have singularities at this position (6.1)? The guess is that they do, but this is not much more than an extrapolation.

Finally, it should perhaps be emphasized that the nature of the singularity of $T_{22}^{(4)}$ at Eq. (6.1) is also expected to change for nonforward directions, just as $T_{22}^{(2)}$ as discussed in Sec. IV.

\section{ACKNOWLEDGMENTS}

The authors would like to thank the Theory Division at CERN for its kind hospitality. This work was supported in part by the U.S. Department of Energy under Grant No. DEFG02-91ER40651, Task B, and under Grant No. DE-FG0284ER40158.
[1] N. N. Khuri and T. T. Wu, preceding paper, Phys. Rev. D 56, 6779 (1997).
[2] K. Symanzik, Prog. Theor. Phys. 20, 690 (1958).

[3] N. N. Khuri, Ann. Phys. (N.Y.) 242, 332 (1995). 